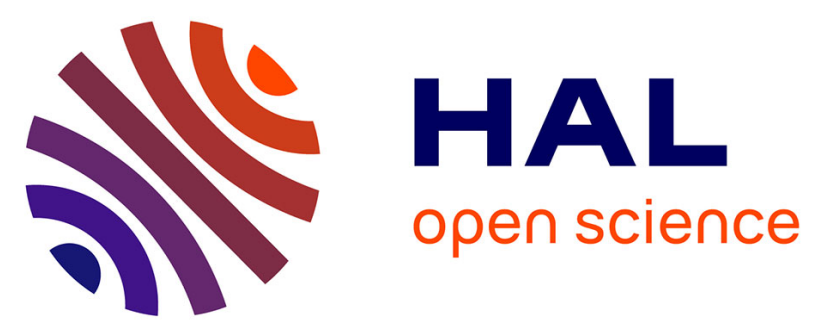

\title{
Photoionization-induced dynamics of the ammonia cation studied by wave packet calculations using curvilinear coordinates
}

Alexandra Viel, Wolfgang Eisfeld, Christian R. Evenhuis, Uwe Manthe

\section{To cite this version:}

Alexandra Viel, Wolfgang Eisfeld, Christian R. Evenhuis, Uwe Manthe. Photoionization-induced dynamics of the ammonia cation studied by wave packet calculations using curvilinear coordinates. Chemical Physics, 2008, 347 (1-3), pp.331-339. 10.1016/j.chemphys.2007.10.001 . hal-01118801

\author{
HAL Id: hal-01118801 \\ https://hal.science/hal-01118801
}

Submitted on 12 May 2020

HAL is a multi-disciplinary open access archive for the deposit and dissemination of scientific research documents, whether they are published or not. The documents may come from teaching and research institutions in France or abroad, or from public or private research centers.
L'archive ouverte pluridisciplinaire HAL, est destinée au dépôt et à la diffusion de documents scientifiques de niveau recherche, publiés ou non, émanant des établissements d'enseignement et de recherche français ou étrangers, des laboratoires publics ou privés. 


\title{
Photoionization-induced dynamics of the ammonia cation studied by wave packet calculations using curvilinear coordinates
}

\author{
Alexandra Viel ${ }^{\mathrm{a}}$ \\ Laboratoire de Physique des Atomes, \\ Lasers, Molécules et Surfaces (PALMS), \\ CNRS UMR 6627 \& Université de Rennes 1, \\ Campus de Beaulieu, F-35042 Rennes, France \\ Wolfgang Eisfeld ${ }^{\mathrm{b}}$, Christian R. Evenhuis, and Uwe Manthe ${ }^{\mathrm{c}}$ \\ Theoretische Chemie, Fakultät für Chemie, Universität Bielefeld, \\ Postfach 100131, D-33501 Bielefeld, Germany
}

(Dated: October 10, 2007)

\begin{abstract}
The determination of the photoelectron spectrum of $\mathrm{NH}_{3}$ and of the internal conversion dynamics of $\mathrm{NH}_{3}^{+}$recently published [J. Chem. Phys. 124, 214396 (2006)] is complemented by the investigation of the effect of the vibrational angular momenta couplings on the dynamics. The multi-configurational time-dependent Hartree method is used to propagate a wave-packet on the analytical anharmonic six-dimensional three-sheeted potential energy surface for the ground and first excited states of the ammonia cation. Curvilinear coordinates and the associated quasi-exact kinetic energy operator suitable for the multi-configurational time-dependent Hartree scheme are employed. A non negligible effect of the use of Cartesian normal modes instead of the curvilinear coordinates is observed on the low energy part of the photoelectron spectrum. However, the three different time scales found in the dynamical calculations for the second absorption band are very similar regardless of the use of either normal modes or curvilinear coordinates.
\end{abstract}

\footnotetext{
a Electronic address: alexandra.viel@univ-rennes1.fr

b Electronic address: wolfgang.eisfeld@uni-bielefeld.de

c Electronic address: uwe.manthe@uni-bielefeld.de
} 


\section{INTRODUCTION}

The photoelectron $(\mathrm{PE})$ spectrum of ammonia $\left(\mathrm{NH}_{3}\right)$ has been of great interest both to experimentalists and theoreticians for a long time. Despite the seeming simplicity of the molecule, the PE spectrum shows several interesting features, which are still not fully understood. The photoionization has been investigated by numerous experimental techniques using He I, He II, x-ray, and synchrotron radiation. ${ }^{1-4}$ The recent study by Edvarsson et $a l .^{5}$ provides high-resolution He I and He II experimental data and also gives an excellent overview of the extended literature.

The two lowest PE bands are due to ionizations out of the non-bonding highest $a_{1}$ orbital and the degenerate set of bonding $e$ orbitals, respectively. The observed spectra for these two ionizations are of completely different character, indicating very different dynamics following the photo process. The first band shows a long and fairly regular, well-resolved progression, which is assigned to be due to the umbrella vibrational mode. The long progression is caused by the fact that the neutral ground state has $C_{3 v}$ pyramidal equilibrium structure whilst the ionic ground state assumes a planar $D_{3 h}$ equilibrium geometry. A much weaker secondary progression is superimposed to these $\nu_{2}$ features, the origin of which has been discussed quite controversially in the literature. ${ }^{5-7}$

By contrast, the second PE band is extremely broad and shows almost no resolved structure. The corresponding first excited state of the cation is a Jahn-Teller (JT) active ${ }^{2} E$ state. It has been shown that the overall shape of this band is largely due to a strong two-mode JT coupling of the asymmetric bending and stretching modes of $e$ symmetry. ${ }^{8}$ A point of considerable interest is the absence of any detectable fluorescence from the excited state even at ionization energies below the known dissociation limit of $\mathrm{NH}_{3}^{+} \cdot{ }^{9}$ This can be interpreted as a strong indication that an ultrafast internal conversion to the ground state may take place, thus quenching the radiative decay. Such ultrafast dynamics may be caused by the presence of a conical intersection between the $\tilde{X}^{2} A_{2}^{\prime \prime}$ and $\tilde{A}^{2} E$ states as proposed already in Ref. 9 and confirmed by self-consistent-field configuration-interaction computations. ${ }^{10}$

A first investigation of the decay dynamics has been performed by Woywod et al. ${ }^{11}$ This study was based on a vibronic coupling model ${ }^{12-14}$ that included only linear coupling terms. It included the coupling of the non-degenerate ground state with the degenerate first excited state known as the pseudo-Jahn-Teller (PJT) effect. ${ }^{12,15-17}$ Indeed, a conical intersection ${ }^{18,19}$ 
between ground and excited state was found in this study and fast but incomplete decay to the ground state was predicted. However, the employed model had significant shortcomings. First, the coordinates used to describe the umbrella motion were chosen inappropriately. Second, the dependence of the PJT coupling on the umbrella angle was modeled incorrectly and did not display the correct symmetry properties. Moreover, the strong anharmonicities present in the system have not been included in the modeling.

An improved modeling of the vibronic coupling, which overcomes the shortcomings of Woywod et al., ${ }^{11}$ has recently been developed and the nonadiabatic dynamics have been studied by means of full-dimensional wave packet propagations. ${ }^{20} \mathrm{~A}$ new set of coupled diabatic PESs was determined based on accurate multireference configuration interaction (MRCI) ab initio calculations. The potential energy matrix representing the diabatic electronic states $^{21-23}$ includes higher order JT and PJT coupling elements ${ }^{24,25}$ and the dependence of the PJT coupling on the umbrella motion is appropriately accounted for. The PESs are expanded in curvilinear symmetry coordinates rather than normal mode coordinates. The dynamical calculations, however, have utilized normal mode coordinates. This study yielded very satisfactory results for both spectral bands discussed. However, it also showed only incomplete radiationless decay to the ground state within the first $100 \mathrm{fs}$.

The use of normal mode coordinates in the dynamical calculations of the previous study constituted an approximation since a simple diagonal kinetic energy operator had been used, i.e., the vibrational angular momenta present in the full Watson kinetic energy operator had been neglected. Recent work on the $\mathrm{CH}_{3}$ radical has shown that this approximation can result in significant errors if large amplitude motion occurs. Since $\mathrm{NH}_{3}^{+}$ions resulting from ionization of $\mathrm{NH}_{3}$ show even larger displacements than present in the $\mathrm{CH}_{3}$ study of Ref. 26, the present work aims on clarifying the effect. To this end, dynamical calculations employing the curvilinear coordinates of Ref. 26 are performed using the MCTDH approach and compared to the results obtained using normal mode coordinates. The new results obtained employing curvilinear coordinates serve as an exact reference to evaluate the size and relevance of possible errors resulting from the diagonal normal mode approximation to the kinetic energy operator. The effects are discussed for both bands of the $\mathrm{NH}_{3}$ photoionization spectrum. Given the different structures of both bands, these results allow conclusions to be drawn, which could be transferred to several other molecular systems.

The article is organized as follows: Sec. II discusses the details of the $\mathrm{NH}_{3}^{+}$system, the 
curvilinear coordinates used as well as relevant aspects of the dynamical method employed. Detailed results are presented in Sec. III, while Sec. IV gives a thorough discussion of the results, which are briefly summarized in the conclusions (Sec. V) of the work.

\section{THE $\mathrm{NH}_{3}^{+}$SYSTEM AND THE NUMERICAL METHOD}

The study of the photoelectron spectrum of ammonia requires the knowledge of the ground state potential energy surface $(\mathrm{PES})$ of $\mathrm{NH}_{3}$ and of the coupled potential energy surfaces for the ground and the first excited states of $\mathrm{NH}_{3}^{+}$. In this work, we employed the full dimensional potentials we determined in our previous study and described in detail in Ref. 20. Therefore, we will only give a brief outline of the model used and refer the interested reader to the original work.

The analytic representation of the three PESs, corresponding to the $\tilde{X}^{2} A_{2}^{\prime \prime}$ and the $\tilde{A}^{2} E^{\prime}$ states of $\mathrm{NH}_{3}^{+}$at the planar $D_{3 h}$ reference geometry, relies on polynomial expansions of the diabatic potential energy matrix elements. These expansions are defined in terms of symmetry coordinates composed of bond distances and bending angles. The use of symmetry allows to perform a "diabatization by ansatz" of the purely adiabatic state energies obtained from $a b$ initio calculations. The structure of the non-vanishing terms of the diabatic potential energy matrix are determined by the symmetry of the system and the requirement that the total Hamiltonian has to be invariant under the symmetry operations of the molecular point group. The present model contains the higher-order Jahn-Teller and pseudo Jahn-Teller couplings derived earlier ${ }^{24,25}$ in order to account for the strong anharmonicity of the potentials. The effect of the dependence of the PJT coupling strength on the pyramidalization coordinate is included by a simple $a_{2}^{\prime \prime}-e^{\prime}$ mode-mode coupling pattern. Other mode-mode couplings $\left(e^{\prime}-e^{\prime}, a_{1}^{\prime}-e^{\prime}, a_{1}^{\prime}-a_{2}^{\prime \prime}\right)$ are still neglected in the present model. The surface for $\mathrm{NH}_{3}$ has been determined using a similar ansatz only without couplings to other electronic states.

The expansion coefficients of the diabatic potential matrix have been determined by fitting the eigenvalues of the matrix with respect to highly accurate $a b$ initio data. The underlying ab initio points used for the determination of the parameters have been obtained at the MRCI level using Dunning's correlation consistent augmented atomic orbital basis of quadruple-zeta quality. ${ }^{27,28}$ Since MRCI is not size-extensive, the vertical ionization energy 
to the ionic ground state was determined by coupled-cluster calculations at RCCSD(T)/augcc-pVQZ level of theory. The full details of the ab initio calculations are given in Ref. 20.

The dynamics of $\mathrm{NH}_{3}$ after ionization to the ground or the excited electronic state of $\mathrm{NH}_{3}^{+}$ is investigated within a time-dependent formalism by means of wave packet propagations performed within the MCTDH approach. ${ }^{29-31}$ For the present system, the MCTDH wave function reads

$$
\psi\left(q_{i}, q_{2}, \ldots q_{6}, t\right)=\sum_{n_{1}} \cdots \sum_{n_{6}} \sum_{\sigma=1}^{3} A_{n_{1} \cdots n_{6} \sigma}(t) \prod_{i=1}^{6} \phi_{n_{i}}^{(i)}\left(q_{i}, t\right) \chi_{\sigma},
$$

where the $A_{n_{1} \cdots n_{6} \sigma}$ denote the time-dependent expansion coefficients and the $\phi_{n_{i}}^{(i)}$ the singleparticle function in the nuclear coordinates $q_{i} ; i=1, \cdots 6$. The $\chi_{\sigma} ; \sigma=1,2,3$ specify the diabatic electronic states. For the evaluation of the potential energy matrix elements the CDVR scheme ${ }^{20,32}$ has been used and for the integration of the equations of motion a specially adapted integrator ${ }^{33,34}$ has been employed. The efficiency of MCTDH depends crucially on the choice of coordinates. The amount of correlation present in the coordinate system employed determines the number of single-particle function coefficients required to converge the expansion in Eq. 1.

In the previous work, ${ }^{20}$ the six mass-weighted Cartesian normal modes corresponding to vibrational motions and constructed at the equilibrium geometry of the ionic ground state (thus in $\mathrm{D}_{3 h}$ symmetry) were used for the dynamical study. A simple Cartesian kinetic energy operator $\hat{T}=-\frac{1}{2} \sum_{i=1}^{6} \frac{\partial^{2}}{\partial q_{i}^{2}}$ has been used and the vibrational angular momenta present in the Watson kinetic energy operator have been neglected. This choice is efficient and reliable for small displacements.

Very recently, a curvilinear coordinate system for $\mathrm{AB}_{3}$ systems has been proposed, which was specifically designed to facilitate efficient MCTDH calculations. ${ }^{26}$ This coordinate system is employed in the new calculations of the present work. The six internal curvilinear coordinates $\rho, \vartheta, \varphi, \theta, \phi, \chi$ of Ref. 26 are based on the three Radau vectors of the $\mathrm{AB}_{3}$ system. $\rho, \vartheta, \varphi$ correspond to the hyperspherical representation of the three Radau lengths. The umbrella angle $\theta$ is defined as the angle between each of the Radau vectors and the trisector of the Radau vectors. The two angles $\phi$ and $\chi$ specify the projections of the Radau vectors on a plane perpendicular to the trisector. The corresponding quantum mechanical 
kinetic energy operator for $J=0$ reads:

$$
\hat{T}=-\frac{1}{2} g^{-1 / 2}\left(\sum_{i, j=1}^{6} \frac{\partial}{\partial q_{i}} g^{1 / 2} G_{q_{i}, q_{j}} \frac{\partial}{\partial q_{j}}\right)
$$

where the volume element is given by

$$
g^{1 / 2}=4 \sin \vartheta \sin ^{3} \theta \sin \beta / \gamma(\phi, \chi)
$$

and the 41 factorized terms of the non vanishing elements of the $G$ matrix are given in Table I. In Eq. 3 the notation

$$
\gamma(\phi, \chi)=\frac{1}{(\cos \phi-\cos \chi) \sin \phi}
$$

has been introduced. Due to the unfactorized $\gamma(\phi, \chi)$ term, the exact kinetic energy operator (Eq. 2) cannot be represented as a sum of products of one-dimensional operators and therefore does not allow for an efficient implementation in MCTDH approach. Therefore, in Ref 26 a quasi-exact kinetic operator was derived by performing a second order Taylor expansion of $\gamma(\phi, \chi)$ around the $\mathrm{C}_{3 v}$ geometries $(\phi=\pi / 3, \chi=\pi)$

$$
\gamma(\phi, \chi) \approx \gamma_{\mathrm{sec}}(\phi, \chi)=\frac{4}{3 \sqrt{3}}\left[1+\left(\phi-\frac{\pi}{3}\right)^{2}\right]\left[1+\frac{1}{3}(\chi-\pi)^{2}\right]
$$

This quasi-exact kinetic energy operator yielded accurate results when tested in calculations of $\mathrm{CH}_{3}$ vibrational eigenstates. To further improve the accuracy of the kinetic energy operator, in this work an even higher order expansion

$$
\begin{aligned}
\gamma(\phi, \chi) \approx \gamma_{\text {higher }}(\phi, \chi)=\frac{4}{3 \sqrt{3}} & {\left[1+\left(\phi-\frac{\pi}{3}\right)^{2}-\frac{1}{3 \sqrt{3}}\left(\phi-\frac{\pi}{3}\right)^{3}+\frac{3}{4}\left(\phi-\frac{\pi}{3}\right)^{4}\right] } \\
\times & {\left[1+\frac{1}{3}(\chi-\pi)^{2}+\frac{1}{12}(\chi-\pi)^{4}\right], }
\end{aligned}
$$

is also considered, which corresponds to a factorized approximation of the fourth order Taylor expansion of $\gamma$.

\section{RESULTS}

\section{A. ZPE considerations}

The effect of the different dynamical coordinates (curvilinear versus Cartesian normal modes) is first investigated by looking at the zero point energies of $\mathrm{NH}_{3}, \mathrm{NH}_{3}^{+}$and model 
TABLE I: Non vanishing exact $G$ matrix elements of Eq. 2.

\begin{tabular}{|c|c|}
\hline$\rho, \rho$ & 1 \\
\hline$\vartheta, \vartheta$ & $\frac{1}{\rho^{2}}$ \\
\hline$\varphi, \varphi$ & $\frac{1}{\rho^{2} \sin ^{2} \vartheta}$ \\
\hline$\theta, \theta$ & $\begin{array}{l}+\frac{1}{4} B \sin ^{2} \phi \cos ^{2} \chi-\frac{1}{8} C \sin 2 \phi \sin 2 \chi \\
+\frac{1}{4} B \cos ^{2} \phi \sin ^{2} \chi+\frac{1}{4} A \sin ^{2} 2 \phi\end{array}$ \\
\hline$\theta, \phi$ & $\begin{array}{l}-\frac{1}{8} B \cot \theta \sin 2 \phi \sin ^{2} \chi \quad+\frac{1}{8} C \cot \theta \sin ^{2} \phi \sin 2 \chi-\frac{1}{4} B \cot \theta \sin ^{3} \phi \cos \chi \\
+\frac{1}{8} C \cot \theta \sin 2 \phi \sin \phi \sin \chi-\frac{1}{2} A \cot \theta \sin ^{2} \phi \sin 2 \phi\end{array}$ \\
\hline$\theta, \chi$ & $\begin{array}{l}-\frac{1}{8} B \cot \theta \sin 2 \chi\left(1-3 \cos ^{2} \phi\right)-\frac{1}{4} B \cot \theta \sin \chi \cos \phi\left(1+\cos ^{2} \phi\right) \\
+\frac{1}{8} C \cot \theta \sin 2 \phi\left(1-3 \cos ^{2} \chi\right)+\frac{1}{4} C \cot \theta \sin \phi \cos \chi\left(1+\cos ^{2} \phi\right) \\
-\frac{1}{2} A \cot \theta \sin 2 \phi \sin \phi \sin \chi\end{array}$ \\
\hline$\phi, \phi$ & $\begin{array}{ll}-\frac{1}{4} B \sin ^{4} \phi & -\frac{1}{4} B \csc ^{2} \theta \sin 2 \phi \sin \phi \cos \chi \\
-\frac{1}{4} B \sin ^{2} \phi \sin ^{2} \chi+\frac{1}{2} B \cot ^{2} \theta \sin ^{4} \phi \sin ^{2} \phi & -\frac{1}{2} C \cot ^{2} \theta \sin ^{3} \phi \sin \chi\end{array}$ \\
\hline$\phi, \chi$ & $\begin{array}{l}+\frac{1}{4} C \sin ^{2} \phi\left(1+\cos ^{2} \phi\right)+\frac{1}{2} B \cot ^{2} \theta \sin \phi \sin \chi+A \cot ^{2} \theta \sin ^{3} \phi \sin \chi \\
-\frac{1}{4} C \sin 2 \phi \sin \phi \cos \chi-\frac{1}{8} B \cot ^{2} \theta \sin 2 \phi \sin 2 \chi \\
+\frac{1}{4} C\left(1-2 \csc ^{2} \theta\right) \sin ^{2} \phi \sin ^{2} \chi\end{array}$ \\
\hline$\chi, \chi$ & $\begin{array}{c}-\frac{1}{2} B \cot ^{2} \theta\left(1+\cos ^{2} \phi\right) \cos \phi \cos \chi+B \cot ^{2} \theta \cos ^{2} \phi \cos ^{2} \chi+\frac{1}{4} B \cos ^{2} \phi \sin ^{2} \phi \\
-\frac{1}{2} C \cot ^{2} \theta\left(1+\cos ^{2} \phi\right) \sin \chi \sin \phi-B \cot ^{2} \theta \cos \phi \cos \chi+\frac{1}{4} B \sin ^{2} \phi \cos ^{2} \chi \\
+\frac{1}{2} B \cot ^{2} \theta\left(1+\cos ^{2} \phi\right) \quad+\frac{1}{4} C \cot ^{2} \theta \sin 2 \phi \sin 2 \chi-A \sin ^{2} \phi \sin ^{2} \chi \\
+A \csc ^{2} \theta\left(1+\cos ^{2} \phi\right) \sin ^{2} \phi-A \csc ^{2} \theta \sin 2 \phi \sin \phi \cos \chi \\
-\frac{1}{4} B \sin 2 \phi \sin \phi \cos \chi\end{array}$ \\
\hline & 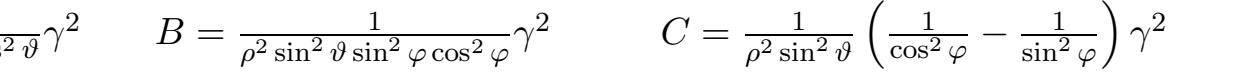 \\
\hline
\end{tabular}

systems. Zero point energies have been determined by propagation in imaginary time using the MCTDH approach. Given the very small value of the ground state tunneling splitting in $\mathrm{NH}_{3}$, no attempt has been made to reach the vibrational ground state of the double well and the ground state energy of the system is approximated by the energy of the localized wavefunction. The energies summarized in Table II have been obtained after an imaginary 
time propagation of 2000 a.u. of an initially optimized Hartree wavepacket. The table

TABLE II: Comparison of the zero point energies $[\mathrm{eV}]$ of $\mathrm{NH}_{3}^{+}, \mathrm{NH}_{3}$ and the model systems obtained using normal modes and the curvilinear coordinates together with two associated kinetic operator using the two expansions of $\gamma(\phi, \chi): \gamma_{\text {higher }}$ (Eq. 6) and $\gamma_{\text {sec }}$ (Eq. 5). For ease of comparison, the last column repports the difference between the normal modes and the quasi-exact curvilinear with the expansion $\gamma_{\text {higher }}$ results.

\begin{tabular}{lcccc}
\hline \hline species & normal modes & curvilinear $\left(\gamma_{\text {higher }}\right)$ & curvilinear $\left(\gamma_{\text {sec }}\right)$ & difference \\
\hline $\mathrm{NH}_{3}^{+}$ & 10.82779 & 10.82814 & 10.82811 & 0.00035 \\
$\mathrm{NH}_{3}$ & 0.58510 & 0.62565 & 0.62561 & 0.04055 \\
$\operatorname{model}[0.00]$ & 0.60991 & 0.60995 & & 0.00004 \\
$\operatorname{model}[-.38]$ & 0.59584 & 0.63461 & & 0.03877 \\
model[-.58] & 0.58413 & 0.67046 & 0.08633 \\
\hline \hline
\end{tabular}

compares the energies obtained using the normal mode description of Ref. 20 and the energies obtained using the curvilinear coordinates and the associated kinetic operators (Eq. 2) with the two different expansions of the $\gamma(\phi, \chi)$ function (Eqs. 5 and 6$)$. The last column shows the difference between computations using the normal mode description and the most accurate calculations using the $\gamma_{\text {higher }}(\phi, \chi)$ expansion. For $\mathrm{NH}_{3}^{+}$, the three dynamical computations give similar zero point energies. This was to be expected since the reference geometry of the normal modes is the $\mathrm{NH}_{3}^{+}$equilibrium geometry. A much larger difference of $0.04 \mathrm{eV}$, which is about 100 times larger than the one computed for $\mathrm{NH}_{3}^{+}$, is obtained for $\mathrm{NH}_{3}$. In order to to gain a deeper insight into the source of this difference, a model system is studied, which corresponds to a $\mathrm{NH}_{3}$ molecule limited to a single well along the umbrella motion. The potential energy surface used for this model is the same as for $\mathrm{NH}_{3}$ except for its dependence on the umbrella coordinate $\mathrm{S}_{2}$ (see ref. 20 for the coordinate definition) for which a simple harmonic well is used. The frequency of this well approximates the physical $\mathrm{NH}_{3}$ system. We varied the position of the minimum of the well from $S_{2}=0$, which corresponds to a planar geometry, to $S_{2}=-0.58$ a.u. The value $S_{2}=-0.38$ a.u. coincides with the true position of the well in $\mathrm{NH}_{3}$. The differences in energy between the curvilinear and the normal mode descriptions are seen to increase rapidly when the equilibrium geometry differs from the reference geometry. 
For both the pyramidalized $\mathrm{NH}_{3}$ and the planar $\mathrm{NH}_{3}^{+}$, the differences in the zero point energies calculated using the two different expansions of $\gamma(\phi, \chi)$ (Eqs. 5, 6) are insignificant.

\section{B. Ionization to the ground state}

The first band of the $\mathrm{NH}_{3}$ ionization spectrum is calculated by propagating the $\mathrm{NH}_{3}$ ground state wave packet vertically shifted to the $\mathrm{NH}_{3}^{+}$ground state PES employing the MCTDH approach. The spectrum, computed by Fourier transformation of the autocorrelation function, is presented in Fig. 1. The basis set used to propagate the wave packet for

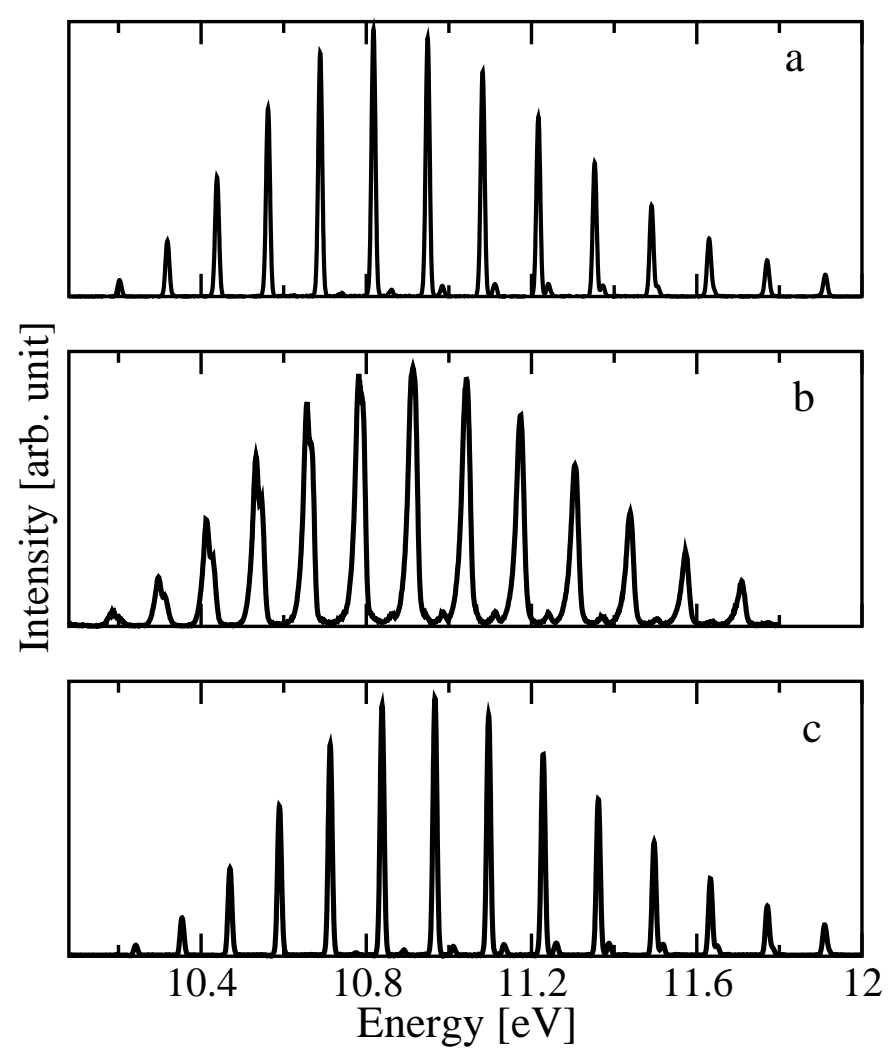

FIG. 1: First band of the photoelectron spectrum of $\mathrm{NH}_{3}$ : present results (top panel), experimental spectrum from Ref. 5 (central panel) and result from Ref. 20 (bottom panel).

$200 \mathrm{fs}$ is given in Table III. A Gaussian damping factor, $\exp \left(-t^{2} / t_{\text {damp }}^{2}\right)$ with $t_{\text {damp }}=200 \mathrm{fs}$, has been employed to avoid spurious oscillations due to the finite propagation time. In Fig. 1, 
TABLE III: Details of the wave packet representation where for each of the coordinates, "range ${ }_{X}$ indicates the limits of the box used [in atomic units], $N_{X}$ the numbers of Fourier grid points, and $n_{X}$ the number of single particle functions used for the propagation after an ionization to the ground state $(X=A)$ and to the excited state $(X=E)$ of $\mathrm{NH}_{3}^{+}$

\begin{tabular}{c|ccr|crc}
\hline \hline coordinate & $\operatorname{range}_{A}$ & $N_{A}$ & $n_{A}$ & $\operatorname{range}_{E}$ & $N_{E}$ & $n_{E}$ \\
\hline$\rho$ & {$[110: 210]$} & 54 & 6 & {$[105: 245]$} & 54 & 7 \\
$\vartheta$ & {$[0.70: 1.2]$} & 32 & 4 & {$[0.65: 1.25]$} & 32 & 5 \\
$\varphi$ & {$[0.55: 1.05]$} & 32 & 4 & {$[0.45: 1.12]$} & 32 & 5 \\
$\theta$ & {$[0.65: 2.49]$} & 64 & 6 & {$[0.43: 2.71]$} & 96 & 15 \\
$\phi$ & {$[0.33: 2.20]$} & 54 & 4 & {$[0.15: 2.28]$} & 64 & 15 \\
$\chi$ & {$[1.74: 4.54]$} & 54 & 4 & {$[1.50: 4.78]$} & 64 & 15 \\
\hline basis size & & 27648 & & 1771875 & \\
\hline \hline
\end{tabular}

we compare the present results (panel a) to the experimental spectra ${ }^{5}$ (panel b) and to the calculations $^{20}$ within the normal mode description (panel c). The theoretical and the experimental results are in good agreement for the main vibrational progression which can be assigned to the umbrella motion. The secondary progression seen in the theoretical spectra are assigned to an excitation of the symmetric stretching mode. The two theoretical spectra are in perfect agreement in the high energy part. At lower energy, however, some noticeable differences are observed. In particular, the 0-0 line position is found to be $10.202 \mathrm{eV}$ when using the curvilinear coordinates while a value of $10.242 \mathrm{eV}$ was found within the normal mode approximation. The experimental line at $10.186 \mathrm{eV}$ is thus reproduced with a significantly better accuracy by the present computation, with an overestimation of only $0.016 \mathrm{eV}$ (an overestimation of $0.056 \mathrm{eV}$ is found within the normal mode approach). The effect on the 0-0 line position is identical to the one reported on the zero point energies in Table II. Given the fact that the quasi-exact kinetic operator has been shown to be accurate within less than $1 \mathrm{~cm}^{-1}$ for the lowest vibrational levels of $\mathrm{CH}_{3},{ }^{26}$ the excellent agreement of the curvilinear computation with the experimental 0-0 line underlines the high accuracy of the $a b$ initio computations, in particular for the ionization energy. Note that in the experimental data, the 0-0 line contains the rotational broadening not present in the theoretical computations. The thorough analysis of the rotational band shape performed by Edvarsson et al. 
indicates that the rovibronic 0-0 transition should correspond to energies slightly higher than the reported peak maxima ( $\sim 5$ to $10 \mathrm{meV})$. This would bring theory and experiment in even closer agreement. At higher energies, the normal mode and the curvilinear studies are in better agreement, probably due to cancellation of errors. However, the deviations from experiment become progressively larger with increasing vibrational excitation. This indicates that the present PES model for the ionic ground state is still insufficient to obtain spectroscopic accuracy for the vibronic levels. This will be discussed in more detail below. The peak positions of experiment and theory are collected in Tab. IV.

TABLE IV: Positions [in eV] of the photoionization lines for the current computation compared to the experimental data ${ }^{5}$ and to the theoretical results using the normal mode coordinates. ${ }^{20}$ Also given are absolute errors between experimental lines and present calculations as well as the corresponding errors in the line spacings $(\Delta$ err.).

\begin{tabular}{cccccc}
\hline \hline assignment & experiment & from Ref. 20 & present results & error & $\Delta$ err. \\
\hline $0-0$ & 10.186 & 10.242 & 10.202 & 0.016 & \\
1 & 10.296 & 10.354 & 10.319 & 0.023 & 0.007 \\
2 & 10.413 & 10.470 & 10.439 & 0.026 & 0.003 \\
3 & 10.532 & 10.590 & 10.562 & 0.030 & 0.004 \\
4 & 10.656 & 10.713 & 10.688 & 0.032 & 0.002 \\
5 & 10.782 & 10.838 & 10.817 & 0.035 & 0.003 \\
6 & 10.911 & 10.966 & 10.949 & 0.038 & 0.003 \\
7 & 11.042 & 11.096 & 11.082 & 0.040 & 0.002 \\
8 & 11.172 & 11.228 & 11.217 & 0.045 & 0.005 \\
9 & 11.305 & 11.362 & 11.353 & 0.048 & 0.003 \\
10 & 11.438 & 11.497 & 11.491 & 0.053 & 0.005 \\
11 & 11.572 & 11.633 & 11.630 & 0.058 & 0.005 \\
12 & 11.708 & 11.771 & 11.770 & 0.062 & 0.004 \\
\hline \hline
\end{tabular}




\section{Ionization to the excited state}

The ionization to the excited state of $\mathrm{NH}_{3}^{+}$leads to a much less structured spectrum shown in Fig. 2. The dynamics calculations were performed in curvilinear coordinates and

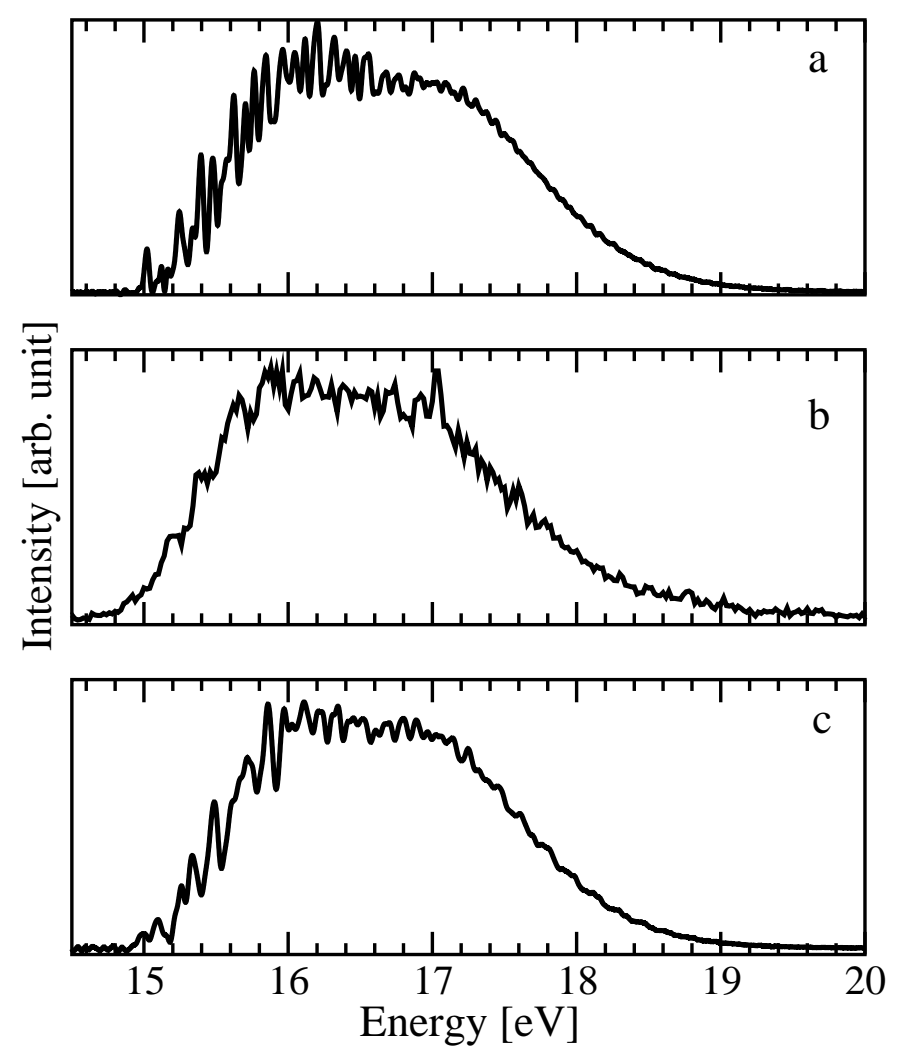

FIG. 2: Second band of the photoelectron spectrum of $\mathrm{NH}_{3}$ : present calculations (top panel), experimental spectrum from Ref. 5 (central panel) and result from Ref. 20(bottom panel).

the spectrum computed from the autocorrelation function up to a time $t=100 \mathrm{fs}$, using $t_{\mathrm{d} a m p}=75 \mathrm{fs}$ for the damping function. The details of the basis set used are summarized in Table III. Like for the lower energy part of the spectral band, the present work is compared to experiment ${ }^{5}$ and to the previous theoretical work using the normal mode description. ${ }^{20}$ Both theoretical computations reproduce the broad and congested spectrum that is observed experimentally. The low energy part of the band appears to be more structured in the theoretical spectra than in the experimental one. This structure is even more pronounced 
when using the curvilinear coordinates. However, the difference to experiment may be due to the choice of the damping time $t_{\mathrm{d} a m p}$ of the autocorrelation function. The broadness of the band is due to efficient vibronic couplings. Importance of the non-adiabatic coupling can be seen in the evolution of the adiabatic populations presented on Fig. 3 up to 100 fs. In the

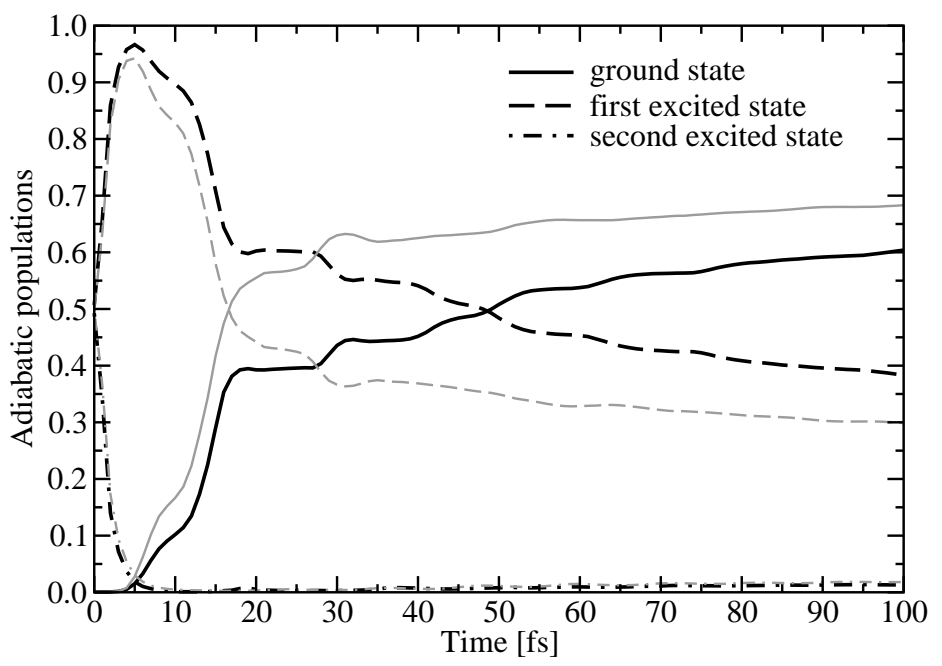

FIG. 3: Adiabatic electronic population of the ground and excited states of $\mathrm{NH}_{3}^{+}$after ionisation to one of the diabatic excited states, as a function of time in fs: the present computations (black lines) are compared with the results from Ref. 20 (grey lines). The basis set size used is given in Table III.

same figure, we report also in grey the results obtained within the normal mode description. ${ }^{20}$ The computed population transfer is qualitatively the same in the two computations. Within $5 \mathrm{fs}$, the highest abiabatic states looses population from 0.5 to nearly 0 , while the middle state sees an increase of its population up to 0.97 . This ultra-fast exchange of population between the two excited states reflects the efficient Jahn-Teller coupling. The population of the ground adiabatic state starts increasing at about 4 fs and exhibits evolution on two time scales. A fast increase up to 0.4 is observed within the first $15-20$ fs followed by a much slower one that is not completed by $100 \mathrm{fs}$. As discussed in the previous work, ${ }^{20}$ the slower second time scale can be interpreted as a dynamical trapping of the wave-packet in the middle adiabatic state. The first time scale can be assigned to the part of the wavepacket finding the conical intersection within the first vibrational period. Quantitatively, the 
results obtained with both the normal modes and the curvilinear coordinates are different after 4-5 fs. After $100 \mathrm{fs}$, the ground state population is found to be 0.60 while it was 0.68 in our previous study. ${ }^{20}$

We want to point out that like in our previous study, ${ }^{20}$ extensive convergence tests have been performed to assure the convergence of the presented results with respect to the MCTDH basis set sizes.

\section{DISCUSSION}

In the present study we investigate the nonadiabatic ionization dynamics of ammonia and the dependence of the corresponding theoretical results on the choice of the coordinate system and kinetic energy operator. Cartesian normal coordinates were used in our previous work together with the simple kinetic energy operator resulting from the neglect of vibrational angular momenta. In this work, we now use a new set of curvilinear coordinates for which the exact kinetic energy operator was derived recently. Only one factor in the non-diagonal matrix representation of the kinetic energy operator is unsuitable for use with the MCTDH method. This factor is approximated by factorized expressions that can be implemented in the MCTDH method. Tests performed in the work by Evenhuis et $a l .{ }^{26}$, showed that the quasi-exact operator used in the present work can be considered to be quantitatively correct. The deviations between second and higher order expansions are well below $1 \mathrm{~cm}^{-1}$ and certainly much smaller than the errors expected from the ab initio calculations and the PES representation.

It turns out that the choice of approximations employed in the normal mode kinetic energy operator have a significant effect on the calculated position of the $0-0$ line for ionization to the cationic ground state. While the ZPE of the cation is only slightly affected, the deviation for the neutral initial state is rather strong $\left(\sim 0.04 \mathrm{eV}\right.$ or $\left.\sim 320 \mathrm{~cm}^{-1}\right)$. The different effect for the two electronic states is readily explained by the fact that the normal coordinates used were determined for the planar $\mathrm{NH}_{3}^{+}$ground state equilibrium geometry while the neural ground state equilibrium geometry is strongly pyramidal. The new calculations yield a 0-0 ionization energy of $10.202 \mathrm{eV}$ compared to the experimental value of $10.186 \mathrm{eV}$. The difference of only $0.016 \mathrm{eV}\left(130 \mathrm{~cm}^{-1}\right)$ demonstrates the high accuracy of the ab initio data. However, taking into account the rotational band shape of the experimental feature, 
the agreement is even better since the peak maximum does not coincide with the $\Delta J=0$ transition but is found at slightly lower energy. This example clearly demonstrates that accurate kinetic energy operators are required when detailed vibrational structures have to be described.

The first band of the PE spectrum is reproduced rather well by the present results as well as by our previous study. Differences between the calculations are only found in the low energy part, which is represented significantly better by the present calculations. However, a closer look reveals that the deviations with respect to experiment get progressively worse at higher energies. At first sight it appears that our model overestimates the anharmonicity. However, the errors of the level spacings (see Table IV) show that there is only a slight and almost constant difference at all energies. There are also some irregularities in the experimental peak positions, which may be due to the circumstance that the peak maxima do not correspond to rotational 0-0 transitions. The largest deviation in level spacings is found for the lowest two peaks and amounts to $0.007 \mathrm{eV}\left(56 \mathrm{~cm}^{-1}\right)$. This result is quite satisfactory, taking into account that the current PES model was developed for the study of the nonadiabatic dynamics and not to represent the vibrational levels to high accuracy. One nevertheless may think about possible improvements. Certainly, the underlying $a b$ initio calculations contribute to the error to some extent. It is not easy to estimate this contribution but with calculations of similar quality it has been possible to predict at least low vibrational levels within a few wavenumbers. ${ }^{35,36}$ Thus, it seems more likely that the major contribution to the errors originates from the analytical representation of the PES. In the current model, mode-mode couplings between the $a_{1}^{\prime}$ and the $a_{2}^{\prime \prime}$ coordinates are completely ignored, which could be responsible for the deviations.

One point of controversy has been the weak secondary progression visible in the experimental and both theoretical spectra of the ionic ground state band. In the thorough study by Edvardsson et al. ${ }^{5}$ this secondary progression was interpreted as being based on a single quantum in the asymmetric bending mode. This assignment was made upon an extrapolation of the level spacings since the first transition of the peak system may be too weak to be observed experimentally or otherwise hidden under the strong transitions. This assignment has its weaknesses and is not supported by our theoretical results. As pointed out by Edvardsson et al., an ionization to a state with a single quantum in the $\nu_{4}$ mode would be Franck-Condon forbidden and thus vibronic coupling to the ${ }^{2} E$ state has to be 
invoked, which, however, vanishes at planarity. In absence of vibronic coupling, excitation of non-totally symmetric modes usually is caused by large differences in the vibrational frequencies. All calculations show that the $\nu_{4}$ frequencies in both neutral and ionic state are very similar and thus the discussed assignment seems rather unlikely. By contrast, the analysis of the time-dependent wave packet dynamics shows that only two vibrational modes are excited upon ionization, $\nu_{1}$ and $\nu_{2}$, the latter very strongly. The excitation of the $\nu_{1}$ mode is of course symmetry allowed and the only weak activity is readily explained by the rather similar $\mathrm{N}-\mathrm{H}$ bond distances in the neutral molecule and the cation ground state. Using a Franck-Condon like argument, the small displacement along the totally symmetric stretching coordinate leads to a large 0-0 FC integral and a small 0-1 integral in $\nu_{1}$. We thus assign the weak secondary features as a progression in the $\nu_{2}$ mode based on a single excitation of the $\nu_{1}$ mode.

The band corresponding to ionization to the first excited ionic state is reproduced very well by the present study as well as by the previous one. The new calculation shows a slightly higher congestion on the red side of the band in good agreement with experiment. However, there is so little detailed information in this spectrum that only very limited conclusions can be drawn from the obtained agreement. As in all previous theoretical studies, the present results show that the congested, largely unstructured shape of the band is the result of ultrafast nonadiabatic dynamics mainly caused by strong JT coupling within the degenerate excited electronic state of the ion.

Though the effect of the kinetic energy operator is rather small on the obtained band shape of the ${ }^{2} E$ PE spectrum, a noticeable change is observed in the nonadiabatic population dynamics. The overall behavior remains the same, however, namely population transfer occurs on three different time scales. This gives additional evidence that the interpretations arrived at in the previous study were correct and are confirmed by the new calculations.

The ultra-fast dynamics following ionization to the excited state is caused by the very strong JT coupling in the ${ }^{2} E$ state and is virtually unchanged by the choice of coordinates. Within roughly 5 fs all population from the upper component of the degenerate ${ }^{2} E$ state is transfered to the lower component. It takes another 10-15 fs for the ground state population to increase to about $40 \%$. This increase is significantly less than found in the previous study. We interpret the rapid increase of ground state population by the wave packet finding the conical intersection reached along the umbrella mode, which connects the 
two lowest electronic states, within one vibrational period. The higher energy components of the wave packet decay immediately through the conical intersection while the lower energy components cannot reach the intersection. The observed differences between Cartesian normal modes and curvilinear coordinates thus reflect the delicate interplay of the motion of the wave packet and the topology of the potential energy surfaces and the intersection hypersurface. The cooled wave packet, which is still trapped on the lower component of the excited state surface, then decays at a slower rate to the ground state. Between 15 and 40 fs the population evolution runs parallel to the previous calculation, including the little hump at about $30 \mathrm{fs}$. After $40 \mathrm{fs}$ the ground state population starts to increase more rapidly when curvilinear coordinates are used. This rate of increase slows down slightly after 60 fs but even at 100 fs, when our propagations where stopped, the ground state population still grows with an almost constant rate. At the end of our propagation time, the ground state population obtained with the new coordinates and the accurate kinetic energy operator is roughly eight percentage points below our previous result and amounts to $60 \%$. This raises the question if these theoretical results can explain the absence of fluorescence observed experimentally. Unfortunately, it is still computationally too demanding to propagate the wave packet for much longer times than reported here. The time it may take for complete decay to the ground state can be roughly estimated by linear extrapolations from our results. Linear regressions of the data between 80 and 100 fs yields times of about 90 ps for completion of the decay, when the present results are used. A comparable estimate based on the data obtained with the normal mode description yields about twice this time. Both would be about an order of magnitude below typical fluorescence life-times. However, the uncertainty of the extrapolation is too large to safely draw any conclusions from this.

\section{CONCLUSIONS}

The new quasi-exact kinetic energy operator results in a slight improvement in details of the calculated photoelectron spectra. Particularly, the $0-0$ transition of the first ionization band is now in astonishing agreement with experiment, which is a result of the more accurate zero-point vibrational energy computations. The well resolved progression of the ionic ground state band is analyzed in detail and is reproduced in very good agreement with experiment. The remaining deviations are attributed to deficiencies in the model for the cou- 
pled potential energy surfaces and the underlying $a b$ initio calculations. The photoelectron band corresponding to the excited ionic state is reproduced equally well by the present and the previous study. However, the corresponding nonadiabatic dynamics following ionization to the excited state shows significant quantitative differences between the two theoretical treatments. The main finding of three different time-scales in the population dynamics and the interpretations of this observation are the same in both studies. By contrast, the numerical results differ noticeably at longer propagation times. Thus, we can conclude that for detailed vibrational structures and sensitive observable approximations in the kinetic energy operators cause differences in quantitative results while leaving the qualitative picture unchanged. When broad spectra resulting from fast nonadiabatic transitions are considered, approximations in the kinetic energy operator are seen to be irrelevant. Resulting differences have been found to be small even at the quantitative level.

Since in the present study the last remaining approximations in the dynamical treatment have been removed, the remaining errors should be due to the electronic structure treatment and the modeling of the diabatic potential energy matrix. The electronic structure calculations cannot be improved easily at reasonable computational cost. By contrast, the potential model leaves room for improvements. Modes corresponding to different vibrational frequencies have not been coupled yet, which could have a significant influence. Another point is that all potentials are constructed for bound state problems and thus dissociation is artificially suppressed. Accounting for dissociation in our model is not trivial but may be necessary. Such further improvements may be addressed in future work.

\section{Acknowledgments}

The authors thank Leif Karlsson for kindly providing the experimental original data files of the two spectral bands published in Ref. 5. The authors acknowledge support via the Sonderforschungsbereich 613 of the Deutsche Forschungsgemeinschaft and a PAI Procope grant.

1 D. W. Turner, C. Baker, and C. R. Brundle, Molecular Photoelectron Spectroscopy (Wiley, London, 1970). 
2 A. W. Potts and W. C. Price, Proc. R. Soc. London Ser. A. 326, 181 (1972).

3 M. S. Banna and D. A. Shirley, J. Chem. Phys. 63, 4759 (1975).

4 M. N. Piancastelli, C. Cauletti, and M. Y. Adam, J. Chem. Phys. 87, 1982 (1987).

5 D. Edvardsson, P. Baltzer, L. Karlsson, B. Wannberg, D. M. P. Holland, D. A. Shaw, and E. E. Rennie, J. Phys. B 32, 2583 (1999).

6 H. Ågren, I. Reineck, H. Veenhuizen, R. Maripuu, R. Arneberg, and L. Karlson, Mol. Phys. 45, 477 (1982).

7 P. Botschwina, in Ion and Cluster Ion Spectroscopy and Structure, edited by J. P. Maier (Elsevier, Amsterdam, 1989).

8 E. Haller, L. S. Cederbaum, W. Domcke, and H. Köppel, Chem. Phys. Letters 72, 427 (1980).

9 G. Dujardin and S. Leach, Can. J. Chem. 63, 1386 (1985).

10 C. Krier, M. T. Praet, and J. C. Lorquet, J. Chem. Phys. 82, 4073 (1985).

11 C. Woywod, S. Scharfe, R. P. Krawczyk, and W. Domcke, J. Chem. Phys. 118, 5880 (2003).

12 H. Köppel, W. Domcke, and L. S. Cederbaum, Adv. Chem. Phys. 57, 59 (1984).

13 H. C. Longuet-Higgins, Adv. Spectrosc. 2, 429 (1961).

14 I. B. Bersuker and V. Z. Polinger, Vibronic Interactions in Molecules and Crystals (Springer, Berlin, 1989).

15 M. H. Perrin and M. Gouterman, J. Chem. Phys. 46, 1019 (1967).

16 J. H. van der Waals, A. M. D. Berghuis, and M. S. de Groot, Mol. Phys. 13, 301 (1967).

17 M. Z. Zgierski and M. Pawlikowski, J. Chem. Phys. 70, 3444 (1979).

18 G. Herzberg and H. C. Longuet-Higgins, Faraday Discussions 35, 77 (1963).

19 W. Domcke, D. R. Yarkony, and H. Köppel, Conical Intersections: Electronic Structure, Dynamics and Spectroscopy (World Scientific, Singapore, 2003).

20 A. Viel, W. Eisfeld, S. Neumann, W. Domcke, and U. Manthe, J. Chem. Phys. 124, 214306 (2006).

21 W. Lichten, Phys. Rev. 229, 131 (1963).

22 F. T. Smith, Phys. Rev. 179, 111 (1969).

23 M. Baer, Chem. Phys. Letters 35, 112 (1975).

24 A. Viel and W. Eisfeld, J. Chem. Phys. 120, 4603 (2004).

25 W. Eisfeld and A. Viel, J. Chem. Phys. 122, 204317 (2005).

26 C. Evenhuis, G. Nyman, and U. Manthe, J. Chem. Phys. p. submitted (2007). 
27 T. H. Dunning, Jr., J. Chem. Phys. 90, 1007 (1989).

28 R. A. Kendall, T. H. Dunning, Jr., and R. J. Harrison, J. Chem. Phys. 96, 6769 (1992).

29 H. D. Meyer, U. Manthe, and L. S. Cederbaum, Chem. Phys. Letters 165, 73 (1990).

30 U. Manthe, H. D. Meyer, and L. S. Cederbaum, J. Chem. Phys. 97, 3199 (1992).

31 M. H. Beck, A. Jäckle, G. A. Worth, and H. D. Meyer, Phys. Rep. 324, 1 (2000).

32 U. Manthe, J. Chem. Phys. 105, 6989 (1996).

33 M. H. Beck and H. D. Meyer, Z. Phys. D 42, 113 (1997).

34 U. Manthe, Chem. Phys. 329, 168 (2006).

35 M. Wyss, E. Riaplov, A. Batalov, J. P. Maier, T. Weber, W. Meyer, and P. Rosmus, J. Chem. Phys. 119, 9703 (2003).

36 T. Rajamäki, A. Milani, and L. Halonen, J. Chem. Phys. 118, 6358 (2003). 